\title{
Optimal cutoff values of waist circumference and the discriminatory performance of other anthropometric indices to detect the clustering of cardiovascular risk factors for metabolic syndrome in Japanese men and women
}

\author{
Kazuyo Nakamura • Hinako Nanri • Megumi Hara Yasuki Higaki Takeshi Imaizumi \\ Naoto Taguchi $\cdot$ Tatsuhiko Sakamoto $\cdot$ Mikako Horita $\cdot$ Koichi Shinchi $\cdot$ Keitaro Tanaka
}

Received: 4 March 2010/Accepted: 6 July 2010/Published online: 30 July 2010

(C) The Japanese Society for Hygiene 2010

\begin{abstract}
Objective To evaluate the pertinent cutoffs of waist circumference (WC) and the discriminatory performance of other anthropometric indices to detect clustering cardiovascular risk factors for metabolic syndrome (MetS) in Japan, where the current WC cutoffs for MetS $(85 \mathrm{~cm}$ for men and $90 \mathrm{~cm}$ for women) remain controversial.

Methods We analyzed the baseline data from 844 subjects (330 men and 514 women) aged 40-69 years who participated in a cohort study in Saga city, Japan, between November 2005 and December 2007. Receiver operating characteristic (ROC) analyses were performed to find an appropriate cutoff (defined as the point nearest to the upper left corner of the ROC curve) of each anthropometric index for the presence of multiple risk factors among dyslipidemia, hypertension, and hyperglycemia [which was defined as hemoglobin A1c (HbA1c) levels at and above 5.2, 5.5, or $5.8 \%$, values approximately corresponding to fasting
\end{abstract}

K. Nakamura $(\bowtie) \cdot$ H. Nanri - M. Hara - T. Imaizumi ·

N. Taguchi $\cdot$ M. Horita $\cdot$ K. Tanaka

Department of Preventive Medicine, Faculty of Medicine,

Saga University, 5-1-1 Nabeshima, Saga 849-8501, Japan

e-mail: 07623003@edu.cc.saga-u.ac.jp

\section{Y. Higaki}

Laboratory of Exercise Physiology, Faculty of Sports and Health

Science, Fukuoka University, Fukuoka, Japan

T. Sakamoto

Asakura Health Welfare Environment Office,

Fukuoka Prefectural Government, Asakura, Japan

K. Shinchi

Division of International Health and Nursing,

Faculty of Medicine, Saga University, Saga, Japan plasma glucose levels of 100,110 , and $120 \mathrm{mg} / \mathrm{dL}$, respectively].

Results The optimal WC cutoff was $88 \mathrm{~cm}$ (sensitivity $60 \%$, specificity $70 \%$ ) for men and $82 \mathrm{~cm}$ (sensitivity $78 \%$, specificity $62 \%$ ) for women; changing the HbA1c cutoff affected the results in women only $(\sim 85 \mathrm{~cm})$. For the currently defined WC cutoffs in Japan, specificity was low $(53-57 \%)$ in men, whereas sensitivity was very low $(32-42 \%)$ in women. Body mass index, proportion of body fat, waist-to-height ratio, and waist-to-hip ratio showed area under the curve values similar to that of WC.

Conclusion The current Japanese criteria of WC for MetS may be low for men and too high and insensitive for women in our study population. Other anthropometric indices such as waist-to-height ratio did not confer an improved discriminatory performance compared with WC.

Keywords Metabolic syndrome - Waist circumference · Cutoff $\cdot$ Cross-sectional study $\cdot$ ROC analysis

\section{Introduction}

The metabolic syndrome (MetS), which was first defined by the World Health Organization in 1998 [1], represents the cluster of metabolically related risk factors for cardiovascular disease (CVD), including abnormal fat distribution, dyslipidemia, hypertension, and hyperglycemia, and the presence of MetS is strongly associated with an increased risk of developing CVD [2]. To define abnormal fat distribution or central obesity, waist circumference (WC) is now regarded as a unique indicator that can identify patients who are at increased risk for obesityrelated CVD, beyond and above the measurement of body mass index (BMI) [3]. However, the selection of the most 
appropriate WC cutoff points appears complex because they are likely to be influenced by sex, race/ethnicity, and other factors [3].

The WC cutoff value for diagnosing MetS among the Japanese remains controversial. In 2005, the Japanese Committee of the Criteria for MetS (JCCMS) decided to adopt WC as an essential component risk factor, and the WC cutoff point was selected as $85 \mathrm{~cm}$ for males and $90 \mathrm{~cm}$ for females $[4,5]$. These cutoff values were determined, in sex-specific linear regression analyses, so as to correspond to a visceral fat area (VFA) of $100 \mathrm{~cm}^{2}$ at which the average number of the component risk factors exceeded 1.0 [6]. However, this VFA was determined without distinction for sex [6] although a recent Japanese study suggested that the optimal cutoff point of VFA differed by sex (132.6 $\mathrm{cm}^{2}$ for men and $91.5 \mathrm{~cm}^{2}$ for women) [7]. Furthermore, some investigators have expressed an additional methodological concern about the above linear regression analyses of the WC cutoff values [8].

On the other hand, several Japanese studies have performed receiver operating characteristic (ROC) analyses for clustering risk factors to examine the optimal WC cutoff point for MetS [7, 9-16]. This is because (1) visceral fat accumulation is now regarded as a key component underlying the cluster of CVD risk factors in MetS [4, 5], and thus (2) the cutoff of WC as an approximate surrogate of visceral fat may be determined as the point that best discriminates the presence or absence of such a cluster, in terms of both sensitivity and specificity. Accordingly, rather different values have been proposed for both Japanese men (82-90 cm) and women (72-83 cm) [7, 9-16]. In 2006, the International Diabetes Federation (IDF) tentatively recommended 90 and $80 \mathrm{~cm}$ for Japanese men and women, respectively, as appropriate cutoffs for diagnosing MetS [2]; however, further investigation of this issue is required.

Another concern regarding WC as a diagnostic component for MetS is its discriminatory performance for the clustering of cardiovascular risk factors, in comparison to other anthropometric indicators. For example, although $\mathrm{WC}$ is considered superior to BMI in this performance [3], WC does not take into account the stature of an individual, and several reports indicate that the waist-to-height ratio may predict the presence of CVD risk factors better than WC [17, 18]. However, the data remain limited on this issue, particularly for the Japanese.

The aims of this study were: (1) to examine the appropriate cutoff value of WC to detect the clustering of CVD risk factors for MetS, and (2) to evaluate the discriminatory performance of other anthropometric indices (i.e., BMI, proportion of body fat, waist-to-height ratio, and waist-tohip ratio) for this clustering, as compared with WC. For both purposes, we conducted ROC analyses using the baseline data of an ongoing cohort study in Japan [19]. Although the current criteria for MetS proposed by worldwide organizations [International Diabetes Foundation (IDF) [2], the American Heart Association/National Heart, Lung, and Blood Institute Scientific Statement [20], and the National Cholesterol Education Program Adult Treatment Panel III (NCEP-ATP III) [21]], as well as the JCCMS [4], adopt fasting plasma glucose (FPG) levels to define hyperglycemia, FPG was not tested in our present baseline survey because the participants were not required to be fasting, for their convenience, as they attended the survey examinations during the daytime. Instead of FPG, we measured hemoglobin A1c (HbA1c), which was employed to define hyperglycemia with changing cutoffs.

\section{Subjects and methods}

\section{Subjects}

In November 2005, we initiated a population-based prospective cohort study in the Saga region, located in the northern part of Kyusyu island, as part of the Japan Multi-Institutional Collaborative Cohort Study (J-MICC Study; Chief investigator: Professor Nobuyuki Hamajima at the Department of Preventive Medicine/Biostatistics and Medical Decision Making, Nagoya University Graduate School of Medicine), which aims at obtaining fundamental data for the prevention of lifestyle-related diseases, mainly cancer, according to genetic traits [22]. The subjects and methods of our cohort study (designated the Saga J-MICC Study) are described in detail elsewhere [19]. Briefly, eligible subjects were all residents of the area corresponding to the former Saga city (the central part of the current Saga city), who were aged 40-69 years at the time of the baseline survey of the Saga J-MICC Study; the former Saga city was administratively consolidated with four adjacent municipalities on October 1, 2005. Candidates were enrolled by investigating the name, sex, date of birth, and address of each resident on the resident register in the city office. A total of 61,447 residents were invited, by mail, to participate in the baseline survey, which was arranged for attendance during the daytime (usually from 9 a.m. to 4 p.m.) between November 1, 2005 and December 22, 2007, in public halls and other available halls near the residential areas of the participants; 12,078 subjects (participation rate $=19.7 \% ; 5082$ men and 6996 women) finally attended the baseline survey examination. Written informed content was obtained from all participants, and the study protocol was approved by the Ethics Committees at Saga University 
Faculty of Medicine and Nagoya University Graduate School of Medicine.

\section{Measurements}

A self-administered questionnaire including queries on current medication and past histories of disease was sent to the participants beforehand, and they were instructed to bring their completed questionnaires to the survey location. At the baseline survey examination, two research nurses double-checked the completed questionnaire of each participant. Systolic blood pressure (SBP) and diastolic blood pressure (DBP) were measured in a sitting position, twice, with an automatic oscillometric sphygmomanometer (BP103iII; Colin Japan, Tokyo, Japan), and the mean value was used for the analysis. The height and weight were measured to the nearest $0.1 \mathrm{~cm}$ and $0.1 \mathrm{~kg}$, respectively, and BMI was calculated as weight $(\mathrm{kg})$ divided by the square of height $(\mathrm{m})$. The proportion of body fat $(\%)$ was examined, with the subjects in bare feet, by a bioimpedance analyzer (BC-500-SV; Tanita, Tokyo, Japan). WC at the umbilicus during the light-exhalation phase and hip circumference at the maximal protuberance of the gluteal region were measured in a standing position, to the nearest $0.1 \mathrm{~cm}$. Waist-to-height and waist-to-hip ratios were calculated as indices of body fat distribution.

\section{Laboratory tests}

Venous blood was drawn from each participant, and serum was separated within $3 \mathrm{~h}$. Serum and whole blood specimens were sent to an external laboratory (SRL, Fukuoka, Japan) to examine serum levels of total cholesterol (TC), high-density lipoprotein (HDL)-cholesterol, and triglyceride (TG), as well as the HbA1c level. TC, HDLcholesterol, and TG were measured enzymatically with an autoanalyzer (7600-300S; Hitachi, Tokyo, Japan), and HbAlc was determined using an immunoassay (Rapidiaauto HbA1c; Fujirebio, Tokyo, Japan) on an autoanalyzer (BM9030; JEOL, Tokyo, Japan). The participants were not required to be in a fasting state at the baseline survey examination, and research nurses recorded, in the questionnaire, the time and day when they had taken the last meal and the time and day of the above blood drawing. In this study, those who had eaten the last meal more than $8 \mathrm{~h}$ before the baseline survey examination were regarded as 'fasting' subjects $(n=1055)$, whereas those who had eaten eight hours or less before the baseline survey examination or had missing data on the time of the last meal were considered as 'nonfasting' subjects $(n=11,023)$ [23]. In this study, only the fasting subjects were employed because the TG level cannot be used to define hypertriglyceridemia for nonfasting subjects.
Statistical analysis

In data analysis, we further excluded subjects who were receiving medication for any of the conditions of hypertension, hyperglycemia, and dyslipidemia $(n=208)$ and those who had a TG level $\geq 1000 \mathrm{mg} / \mathrm{dL}(n=3)$, leaving 844 fasting subjects ( 330 men and 514 women). In this study, an individual was considered to have multiple CVD risk factors if he/she had any two or more of the following (according to the JCCMS criteria [4, 5], if applicable): (1) hypertension, defined as $\mathrm{SBP} \geq 130 \mathrm{mmHg}$ and/or DBP $\geq$ $85 \mathrm{mmHg}$; (2) dyslipidemia, defined as HDL-cholesterol $<40 \mathrm{mg} / \mathrm{dL}$ and/or TG $\geq 150 \mathrm{mg} / \mathrm{dL}$; and (3) hyperglycemia, defined as HbAlc (\%) equal to or larger than each of the following cutoffs: 5.8, 5.5, and 5.2; these values approximately correspond to FPG levels of 120, 110, and $100 \mathrm{mg} / \mathrm{dL}$, respectively, according to Ito et al. [24].

In the ROC analysis, we considered the presence or absence of multiple CVD risk factors as an outcome variable and each anthropometric measurement (i.e., WC, waist-to-height ratio, waist-to-hip ratio, BMI, or proportion of body fat) as a testing variable. The distance between each point on the ROC curve and the upper left corner was calculated as the square root of $\left[(1-\text { sensitivity })^{2}+(1-\right.$ specificity $\left.)^{2}\right]$, and the point with the shortest distance was judged to correspond to the optimal cutoff value $[25,26]$. Furthermore, we calculated the area under the curve (AUC) and its 95\% confidence interval (CI) for each anthropometric measurement and compared AUCs for different measurements by the $\chi^{2}$ test. Statistical analyses were conducted with the SAS (Version 9.1 for Windows; SAS Institute, Cary, NC, USA) and STATA (Version 8.2; Stata, College Station, TX, USA) statistical software packages. A two-sided $P$ value $<0.05$ was considered to be statistically significant.

\section{Results}

Table 1 shows the basic characteristics of the study subjects. The average age was 52.5 years for men and 52.9 years for women. Women showed significantly higher proportions of body fat, and significantly higher TC and HDL-cholesterol levels, whereas men had significantly higher values for height, weight, WC, hip circumference, BMI, waist-to-hip ratio, SBP, DBP, and TG. When the HbA1c cutoff of 5.5\% was arbitrarily used to define hyperglycemia, $34.2 \%$ of men and $16.5 \%$ of women had any two or more CVD risk factors.

Table 2 and Fig. 1 present the results from the ROC analyses for WC in detecting the presence of multiple CVD risk factors. In these analyses, three HbAlc cutoffs (5.8, 5.5 , and $5.2 \%$ ) were used to define hyperglycemia. In men, 
Table 1 Characteristics of study subjects
Values are shown as means \pm standard deviation for continuous variables or numbers (percentages) for prevalences

${ }^{a}$ Defined as systolic blood pressure $\geq 130 \mathrm{mmHg}$ and/or diastolic blood pressure $\geq 85 \mathrm{mmHg}$

b Defined as high-density lipoprotein (HDL)cholesterol $<40 \mathrm{mg} / \mathrm{dL}$ and/or triglyceride $\geq 150 \mathrm{mg} / \mathrm{dL}$

c Defined as hemoglobin $\mathrm{A} 1 \mathrm{c} \geq 5.5 \%$

\begin{tabular}{|c|c|c|c|}
\hline Factor & $\operatorname{Men}(n=330)$ & Women $(n=514)$ & $P$ \\
\hline Age (years) & $52.5 \pm 8.3$ & $52.9 \pm 8.1$ & 0.39 \\
\hline \multicolumn{4}{|l|}{ Age group, $n(\%)$} \\
\hline 40-49 years & $133(40.3)$ & $199(38.7)$ & \multirow[t]{3}{*}{0.85} \\
\hline $50-59$ years & $123(37.3)$ & $201(39.1)$ & \\
\hline $60-69$ years & $74(22.4)$ & $114(22.2)$ & \\
\hline Height (cm) & $168.0 \pm 6.2$ & $155.5 \pm 5.5$ & $<0.01$ \\
\hline Weight (kg) & $66.3 \pm 10.7$ & $53.9 \pm 8.7$ & $<0.01$ \\
\hline Waist circumference $(\mathrm{cm})$ & $85.6 \pm 8.8$ & $80.4 \pm 9.7$ & $<0.01$ \\
\hline Hip circumference $(\mathrm{cm})$ & $92.9 \pm 5.7$ & $90.2 \pm 6.0$ & $<0.01$ \\
\hline Body mass index $\left(\mathrm{kg} / \mathrm{m}^{2}\right)$ & $23.4 \pm 3.3$ & $22.3 \pm 3.5$ & $<0.01$ \\
\hline Body fat proportion (\%) & $22.8 \pm 4.3$ & $32.1 \pm 5.8$ & $<0.01$ \\
\hline Waist-to-height ratio & $0.50 \pm 0.05$ & $0.52 \pm 0.07$ & 0.06 \\
\hline Waist-to-hip ratio & $0.91 \pm 0.05$ & $0.89 \pm 0.08$ & $<0.01$ \\
\hline Systolic blood pressure (mmHg) & $134.3 \pm 18.9$ & $128.2 \pm 20.9$ & $<0.01$ \\
\hline Diastolic blood pressure (mmHg) & $84.8 \pm 11.9$ & $77.1 \pm 12.6$ & $<0.01$ \\
\hline Total cholesterol (mg/dL) & $212.3 \pm 32.9$ & $221.3 \pm 35.1$ & $<0.01$ \\
\hline HDL-cholesterol (mg/dL) & $55.5 \pm 14.9$ & $65.4 \pm 14.3$ & $<0.01$ \\
\hline Triglyceride (mg/dL) & $147.9 \pm 107.7$ & $98.1 \pm 66.6$ & $<0.01$ \\
\hline Hemoglobin A1c (\%) & $5.2 \pm 0.8$ & $5.1 \pm 0.7$ & 0.14 \\
\hline Hypertension $^{\mathrm{a}}, n(\%)$ & $200(60.6)$ & $228(44.4)$ & $<0.01$ \\
\hline Dyslipidemia $^{\mathrm{b}}, n(\%)$ & $141(42.7)$ & $73(14.2)$ & $<0.01$ \\
\hline Hyperglycemia $^{\mathrm{c}}, n(\%)$ & $52(15.8)$ & $76(14.8)$ & 0.72 \\
\hline Any two risk factors, $n(\%)$ & $99(30.0)$ & $74(14.4)$ & $<0.01$ \\
\hline Three risk factors, $n(\%)$ & $14(4.2)$ & $11(2.1)$ & 0.08 \\
\hline
\end{tabular}

the optimal cutoff point of WC with the shortest distance was $88 \mathrm{~cm}$ for all three HbA1c cutoffs, with the sensitivity and specificity ranging from 58 to 63 and from 69 to $75 \%$, respectively. In women, the corresponding cutoff point was $85 \mathrm{~cm}$ for the HbA1c cutoff of $5.8 \%$ (sensitivity $=68 \%$, specificity $=71 \%$ ) and $82 \mathrm{~cm}$ for the cutoff points of $5.5 \%$ (sensitivity $=78 \%$, specificity $=62 \%$ ) and $5.2 \%$ (sensitivity $=72 \%$, specificity $=66 \%$ ). When the current $\mathrm{WC}$ cutoff values defined by the JCCMS $(85 \mathrm{~cm}$ for men and $90 \mathrm{~cm}$ for women) were applied, the respective ranges of the sensitivity and specificity were $69-75$ and $53-57 \%$ for men, in contrast to $32-42$ and $88-90 \%$ for women. As shown in Fig. 1, the AUCs for different HbA1c cutoffs showed similar values in both men (range 0.69-0.71) and women (range 0.73-0.76). Although the AUCs in women appeared larger than the corresponding AUCs in men, the sex differences were not statistically significant $(P$ values for $\mathrm{HbA1c}$ cutoffs of 5.8, 5.5, and 5.2\%: $0.30,0.10$, and 0.50 , respectively).

Table 3 demonstrates the proportions of true positive (TP), false negative (FN), false positive (FP), and true negative (TN) results when the WC cutoff points proposed by the JCCMS, IDF, and the present study were applied to the above ROC analyses with an HbA1c level $\geq 5.5 \%$ defined as hyperglycemia. In men, the FP proportion was largest (0.31) according to the JCCMS criteria as compared with the other two criteria, while the FN proportion became largest (0.18) when the IDF criteria were applied. In women, the IDF criteria made the FP proportion largest (0.39), whereas the JCCMS criteria led to the largest FN proportion (0.10), which much exceeded the corresponding TP proportion (0.06). The WC cutoffs identified in this study provided an intermediate FP or FN proportion in both men and women.

Table 4 shows the comparison of the AUCs and optimal cutoff values for WC, BMI, proportion of body fat, waistto-height-ratio, and waist-to-hip ratio in detecting the clustering of CVD risk factors. Because changing the HbA1c cutoffs gave similar results, the cutoff of $5.5 \%$ was arbitrarily used to define hyperglycemia. The AUCs for the five measurements were not significantly different in either men $(P=0.46)$ or women $(P=0.35)$. The respective optimal cutoff values in men and women were 22.9 and $22.6 \mathrm{~kg} / \mathrm{m}^{2}$ for BMI, 23.2 and $33.9 \%$ for the proportion of body fat, 0.52 and 0.54 for waist-to-height ratio, and 0.92 and 0.89 for waist-to-hip ratio. 
Table 2 Receiver operating characteristic (ROC) analysis of waist circumference (WC) in detecting the presence of multiple cardiovascular risk factors

\begin{tabular}{|c|c|c|c|c|c|c|c|c|c|}
\hline \multirow[t]{3}{*}{ WC cutoff $(\mathrm{cm})$} & \multicolumn{9}{|c|}{ Hyperglycemia defined as } \\
\hline & \multicolumn{3}{|c|}{ Hemoglobin A1c $\geq 5.8 \%$} & \multicolumn{3}{|c|}{ Hemoglobin A1c $\geq 5.5 \%$} & \multicolumn{3}{|c|}{ Hemoglobin A1c $\geq 5.2 \%$} \\
\hline & Sens & Spec & Distance $^{\mathrm{a}}$ & Sens & Spec & Distance $^{\mathrm{a}}$ & Sens & Spec & Distance $^{\mathrm{a}}$ \\
\hline \multicolumn{10}{|l|}{$\operatorname{Men}(n=330)$} \\
\hline 83 & 0.85 & 0.44 & 0.576 & 0.81 & 0.44 & 0.593 & 0.80 & 0.49 & 0.547 \\
\hline 84 & 0.83 & 0.48 & 0.545 & 0.79 & 0.48 & 0.560 & 0.76 & 0.52 & 0.537 \\
\hline 85 & 0.75 & 0.53 & 0.533 & 0.72 & 0.53 & 0.547 & 0.69 & 0.57 & 0.535 \\
\hline 86 & 0.70 & 0.58 & 0.516 & 0.67 & 0.59 & 0.527 & 0.66 & 0.63 & 0.503 \\
\hline 87 & 0.67 & 0.62 & 0.505 & 0.65 & 0.63 & 0.513 & 0.62 & 0.67 & 0.499 \\
\hline 88 & 0.63 & 0.69 & 0.486 & 0.60 & 0.70 & 0.500 & 0.58 & 0.75 & 0.493 \\
\hline 89 & 0.55 & 0.72 & 0.536 & 0.53 & 0.72 & 0.544 & 0.51 & 0.77 & 0.539 \\
\hline 90 & 0.51 & 0.75 & 0.556 & 0.49 & 0.75 & 0.570 & 0.48 & 0.80 & 0.556 \\
\hline \multicolumn{10}{|c|}{ Women $(n=514)$} \\
\hline 80 & 0.84 & 0.52 & 0.511 & 0.85 & 0.54 & 0.488 & 0.78 & 0.56 & 0.490 \\
\hline 81 & 0.82 & 0.56 & 0.471 & 0.84 & 0.59 & 0.444 & 0.77 & 0.62 & 0.449 \\
\hline 82 & 0.77 & 0.60 & 0.456 & 0.78 & 0.62 & 0.437 & 0.72 & 0.66 & 0.442 \\
\hline 83 & 0.71 & 0.65 & 0.458 & 0.68 & 0.66 & 0.465 & 0.64 & 0.69 & 0.477 \\
\hline 84 & 0.68 & 0.67 & 0.461 & 0.66 & 0.69 & 0.464 & 0.60 & 0.71 & 0.494 \\
\hline 85 & 0.68 & 0.71 & 0.434 & 0.65 & 0.72 & 0.447 & 0.56 & 0.74 & 0.506 \\
\hline 86 & 0.60 & 0.75 & 0.473 & 0.58 & 0.77 & 0.483 & 0.50 & 0.78 & 0.549 \\
\hline 87 & 0.55 & 0.79 & 0.499 & 0.52 & 0.80 & 0.522 & 0.44 & 0.81 & 0.587 \\
\hline 88 & 0.50 & 0.83 & 0.529 & 0.48 & 0.84 & 0.541 & 0.40 & 0.85 & 0.619 \\
\hline 89 & 0.44 & 0.85 & 0.583 & 0.41 & 0.86 & 0.604 & 0.35 & 0.88 & 0.666 \\
\hline 90 & 0.42 & 0.88 & 0.594 & 0.38 & 0.88 & 0.634 & 0.32 & 0.90 & 0.692 \\
\hline
\end{tabular}

Presence of any two or more of hypertension (systolic blood pressure $\geq 130 \mathrm{mmHg}$ and/or diastolic blood pressure $\geq 85 \mathrm{mmHg}$ ), dyslipidemia (HDL-cholesterol $<40 \mathrm{mg} / \mathrm{dL}$ and/or triglyceride $\geq 150 \mathrm{mg} / \mathrm{dL}$ ), and hyperglycemia (defined as above). The optimal cutoff values are indicated by bold figures

Sens sensitivity, Spec specificity

a The distance between each point on the ROC curve and the upper left corner

\section{Discussion}

The current WC cutoff values defined by the JCCMS ( $85 \mathrm{~cm}$ for men and $90 \mathrm{~cm}$ for women) are unique in that they have been determined from VFA evaluated by computed tomography [4-6]. However, there remain methodological concerns about the above cutoffs [7, 8], and according to ROC analyses for clustering CVD risk factors in Japanese populations [7, 9-16], the optimal WC cutoff ranged from 82 to $90 \mathrm{~cm}$ for men and from 72 to $83 \mathrm{~cm}$ for women, indicating that the current cutoff might be problematic, particularly for women. In the present study, we found $88 \mathrm{~cm}$ for men and 82 or $85 \mathrm{~cm}$ for women to be appropriate cutoffs. These values were analogous to the results from the above ROC analyses [7, 9-16], but were discordant with the cutoffs defined by the JCCMS, particularly for women $[4,5]$. Under the JCCMS criteria for WC, the specificity for the presence of multiple CVD risk factors in the present study was low for men (53-57\%), and the corresponding sensitivity was very low and perhaps unacceptable for women $(32-42 \%)$. On the other hand, when the IDF criteria $(90 \mathrm{~cm}$ for men and $80 \mathrm{~cm}$ for women) were applied to the present results, the sensitivity in men $(48-51 \%)$ and the specificity in women (52-56\%) turned out to be low.

Even in the ROC analyses using the same approach on this topic, there have been substantial discrepancies regarding the optimal WC cutoff for the Japanese [7, 916]; these discrepancies may have occurred for the following reasons: (1) different [10, 11] or ambiguous [9] measuring sites for WC in earlier studies, as compared with the umbilical site in recent studies [7, 12-16]; (2) different age distributions (e.g., the inclusion of relatively young subjects aged $<40$ years [9-11, 13-15] or elderly subjects aged $\geq 70$ years $[9,11,13]$ ); and (3) different methods in determining the cutoff point (i.e., maximizing the sum of sensitivity and specificity or the Youden index [7, 10-12, $15,16]$, equalizing sensitivity with specificity $[9,13]$, or 


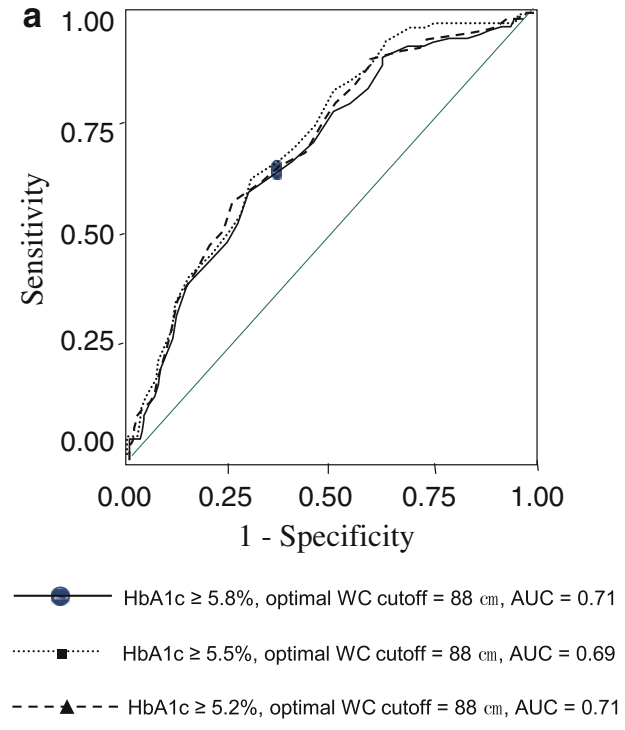

Fig. 1 Receiver operating characteristic curves for waist circumference $(W C)$ in detecting the presence of multiple cardiovascular risk factors for 330 men (a) and 514 women (b). A subject was considered to have multiple risk factors if he/she had any two or more of hypertension (systolic blood pressure $\geq 130 \mathrm{mmHg}$ and/or diastolic

Table 3 Sensitivity (sens), specificity (spec), and proportions of four test results [true positive (TP), false negative (FN), false positive (FP), and true negative $(\mathrm{TN})$ ] for waist circumference (WC) in detecting the

\begin{tabular}{|c|c|c|c|c|c|c|c|}
\hline \multirow[t]{2}{*}{ WC criteria } & \multirow[t]{2}{*}{ WC cutoff $(\mathrm{cm})$} & \multirow[t]{2}{*}{ Sens } & \multirow[t]{2}{*}{ Spec } & \multicolumn{4}{|c|}{ Number (proportion) } \\
\hline & & & & $\mathrm{TP}$ & $\mathrm{FN}$ & FP & $\mathrm{TN}$ \\
\hline \multicolumn{8}{|c|}{ Men $(n=330)$} \\
\hline JCCMS & 85 & 0.72 & 0.53 & $81(0.24)$ & $32(0.10)$ & $101(0.31)$ & $116(0.35)$ \\
\hline This study & 88 & 0.60 & 0.70 & $68(0.21)$ & $45(0.14)$ & $65(0.20)$ & $152(0.46)$ \\
\hline IDF & 90 & 0.49 & 0.75 & $55(0.17)$ & $58(0.18)$ & $53(0.16)$ & $164(0.50)$ \\
\hline \multicolumn{8}{|c|}{ Women $(n=514)$} \\
\hline IDF & 80 & 0.85 & 0.54 & $72(0.14)$ & $13(0.03)$ & $199(0.39)$ & $230(0.45)$ \\
\hline This study & 82 & 0.78 & 0.62 & $66(0.13)$ & $19(0.04)$ & $161(0.31)$ & $268(0.52)$ \\
\hline JCCMS & 90 & 0.38 & 0.88 & $32(0.06)$ & $53(0.10)$ & $50(0.10)$ & $379(0.74)$ \\
\hline
\end{tabular}

JCCMS The Japanese Committee of the Criteria for Metabolic Syndrome, IDF The International Diabetes Federation

Presence of any two or more of hypertension (systolic blood pressure $\geq 130 \mathrm{mmHg}$ and/or diastolic blood pressure $\geq 85 \mathrm{mmHg}$ ), dyslipidemia (HDL-cholesterol $<40 \mathrm{mg} / \mathrm{dL}$ and/or triglyceride $\geq 150 \mathrm{mg} / \mathrm{dL}$ ), and hyperglycemia (hemoglobin A1c $\geq 5.5 \%$ )

attaining about $80 \%$ sensitivity with maximal specificity [14]). Regarding the last point, we selected the cutoff closest to the upper left corner of the ROC curve, and the results remained almost unchanged when we chose the cutoff maximizing sensitivity plus specificity; we did not seek a cutoff with a high sensitivity (e.g., 80\%) at the expense of a low specificity (e.g., $<50 \%$ ). The cutoff selection also should take into account the epidemiologic situation (e.g., the prevalence of an outcome of interest in the target population) and the relative consequence (i.e., cost or benefit) of each test result (i.e., TP, TN, FP, or FN). In the above ROC analyses, however, it seems difficult to

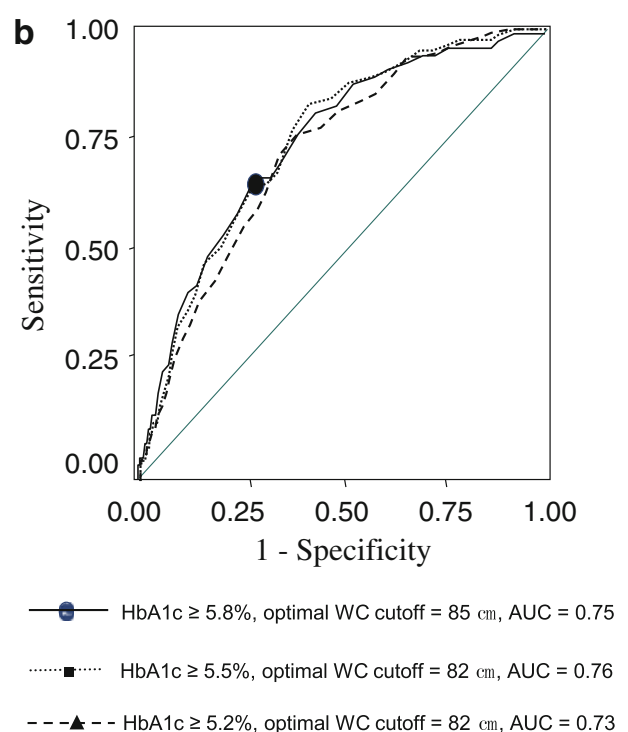

blood pressure $85 \mathrm{mmHg}$ ), dyslipidemia (high-density lipoprotein [HDL]-cholesterol $<40 \mathrm{mg} / \mathrm{dL}$ and/or triglyceride $\geq 150 \mathrm{mg} / \mathrm{dL}$ ), and hyperglycemia [hemoglobin A1c (HbAlc) equal to or larger than each of the following cutoffs: 5.8, 5.5, and 5.2\%]. AUC area under the curve

presence of multiple cardiovascular risk factors according to three different criteria (JCCMS, IDF, and this study)

Number (proportion)

. 
Table 4 Comparison of the areas under the receiver operating characteristic curves (AUCs) and optimal cutoff values for anthropometric indices in detecting the presence of multiple cardiovascular risk factors

\begin{tabular}{|c|c|c|c|c|c|}
\hline Anthropometric index & $\operatorname{AUC}^{\mathrm{a}}(95 \% \mathrm{CI})$ & Optimal cutoff & Sens & Spec & Distance $^{\mathrm{b}}$ \\
\hline \multicolumn{6}{|l|}{$\operatorname{Men}(n=330)$} \\
\hline Waist circumference & $0.69(0.63-0.75)$ & $88 \mathrm{~cm}$ & 0.60 & 0.70 & 0.50 \\
\hline Body mass index & $0.68(0.63-0.74)$ & $22.9 \mathrm{~kg} / \mathrm{m}^{2}$ & 0.78 & 0.56 & 0.50 \\
\hline Body fat proportion & $0.66(0.60-0.72)$ & $23.2 \%$ & 0.65 & 0.59 & 0.54 \\
\hline Waist-to-height ratio & $0.68(0.62-0.74)$ & 0.52 & 0.58 & 0.68 & 0.53 \\
\hline Waist-to-hip ratio & $0.67(0.61-0.73)$ & 0.92 & 0.66 & 0.61 & 0.52 \\
\hline \multicolumn{6}{|l|}{ Women $(n=514)$} \\
\hline Waist circumference & $0.76(0.70-0.81)$ & $82 \mathrm{~cm}$ & 0.78 & 0.62 & 0.44 \\
\hline Body mass index & $0.76(0.70-0.81)$ & $22.6 \mathrm{~kg} / \mathrm{m}^{2}$ & 0.75 & 0.69 & 0.40 \\
\hline Body fat proportion & $0.76(0.70-0.82)$ & $33.9 \%$ & 0.72 & 0.70 & 0.41 \\
\hline Waist-to-height ratio & $0.75(0.70-0.80)$ & 0.54 & 0.67 & 0.70 & 0.44 \\
\hline Waist-to-hip ratio & $0.72(0.67-0.77)$ & 0.89 & 0.73 & 0.59 & 0.49 \\
\hline
\end{tabular}

Presence of any two or more of hypertension (systolic blood pressure $\geq 130 \mathrm{mmHg}$ and/or diastolic blood pressure $\geq 85 \mathrm{mmHg}$ ), dyslipidemia (HDL-cholesterol $<40 \mathrm{mg} / \mathrm{dL}$ and/or triglyceride $\geq 150 \mathrm{mg} / \mathrm{dL}$ ), and hyperglycemia (hemoglobin A1c $\geq 5.5 \%$ )

Sens sensitivity, Spec specificity, $C I$ confidence interval

${ }^{a}$ The AUCs for the five measurements were not significantly different in either men $(P=0.46)$ or women $(P=0.35)$

b The distance between each point on the ROC curve and the upper left corner

other than the Japanese, such as the Mongolians [10], Koreans [27, 28], and Chinese [25], have reported cutoff values (80-92 cm for men and 78-84 cm for women) that were frequently different from those proposed by the IDF, and the IDF criteria may need to be reexamined for such populations as well.

At present, all criteria for MetS adopt FPG to define hyperglycemia (FPG $\geq 110 \mathrm{mg} / \mathrm{dL}$ by NCEP-ATP III [21] and JCCMS [4, 5]; FPG $\geq 100 \mathrm{mg} / \mathrm{dL}$ by the IDF [2] and the American Heart Association/National Heart, Lung, and Blood Institute Scientific Statement [20]). In the present study, we tested HbA1c instead of FPG due to the practical difficulty of requesting participants to maintain their fasting state. In a previous Japanese study, an FPG level $\geq 110 \mathrm{mg} / \mathrm{dL}$ approximately corresponded to an $\mathrm{HbAlc}$ level $\geq 5.5 \%$ [24], and so the arbitrary definition of hyperglycemia $(\mathrm{HbA} 1 \mathrm{c} \geq 5.5 \%)$ employed in most analyses in the present study appears proper. We also changed the HbA1c cutoff to 5.8 or $5.2 \%$, but the results remained similar. A recent cohort study suggested that glycated hemoglobin was more strongly associated with CVD risks than fasting glucose [29], and in this context, our findings might provide useful information for future studies.

In the present study, as compared with $\mathrm{WC}$, other anthropometric indices (e.g., waist-to-height ratio) did not confer an improved performance (i.e., wider AUC) for discriminating the presence or absence of multiple CVD risk factors. Two cross-sectional studies in Asia (in Taiwan [18] and Hong Kong [17]) showed that the waist-to-height ratio predicted the presence of CVD risk factors better than
WC and other anthropometric indices, although statistically significant differences in AUC between such indices were not definitely tested. A few Japanese studies have also indicated a similar superior predictive performance of waist-to-height ratio over waist-to-hip ratio, BMI, and other measurements [30-32]. However, a Dutch study revealed that height did not importantly influence the differences in measures of adiposity or intraabdominal fat volume in women, or intraabdominal fat area in both sexes [33]. Our results did not support the importance of considering height in addition to WC in detecting the cluster of CVD risk factors.

On the other hand, it was unexpected that, in this study, the discriminatory performance of WC was virtually the same as that of BMI. This was partly because WC was highly correlated with BMI (Pearson correlation coefficient: 0.89 in men and 0.82 in women). This suggests that BMI can be used as a surrogate of WC to diagnose MetS among the Japanese. Shiwaku et al. [10] and Oka et al. [7] reported similar findings in Japanese populations, and both studies demonstrated that the optimal BMI cutoff values for predicting multiple metabolic disorders were 24 and $23 \mathrm{~kg} / \mathrm{m}^{2}$ for Japanese men and women, respectively. In the present study, the corresponding cutoff was estimated at about $23 \mathrm{~kg} / \mathrm{m}^{2}$ for both men and women. These cutoffs are lower than the BMI cutoff of $25 \mathrm{~kg} / \mathrm{m}^{2}$ for overweight defined by the WHO [34]. In line with these findings, a WHO expert consultation has recently concluded that the proportion of Asian populations with a high risk of type 2 diabetes and CVD is substantial at BMIs lower than $25 \mathrm{~kg} / \mathrm{m}^{2}$ [35]. 
Table 5 Comparison of fasting and nonfasting subjects in terms of sensitivity and specificity of waist circumference (WC) for detecting each of hypertension, low HDL-cholesterol, and hyperglycemia

\begin{tabular}{|c|c|c|c|c|c|c|}
\hline & \multicolumn{3}{|c|}{ Sensitivity of $\mathrm{WC}^{\mathrm{a}}$} & \multicolumn{3}{|c|}{ Specificity of $\mathrm{WC}^{\mathrm{a}}$} \\
\hline & Fasting $^{\mathrm{b}}$ & Nonfasting $^{\mathrm{c}}$ & $P^{\mathrm{d}}$ & Fasting $^{\mathrm{b}}$ & Nonfasting $^{c}$ & $P^{\mathrm{d}}$ \\
\hline \multicolumn{7}{|l|}{ Men } \\
\hline Hypertension $^{\mathrm{e}}$ & $0.48\left(96 / 200^{f}\right)$ & $0.46\left(916 / 1993^{\mathrm{f}}\right)$ & 0.58 & $0.72\left(93 / 130^{\mathrm{g}}\right)$ & $0.71\left(995 / 1393^{\mathrm{g}}\right)$ & 0.98 \\
\hline Low HDL-cholesterol $^{\mathrm{h}}$ & $0.54(19 / 34)$ & $0.58(265 / 454)$ & 0.78 & $0.62(182 / 296)$ & $0.64(1882 / 2932)$ & 0.32 \\
\hline Hyperglycemia $^{\mathrm{i}}$ & $0.51(27 / 52)$ & $0.51(289 / 572)$ & 0.65 & $0.62(172 / 278)$ & $0.63(1788 / 2814)$ & 0.54 \\
\hline \multicolumn{7}{|l|}{ Women } \\
\hline Hypertension $^{\mathrm{e}}$ & $0.61(139 / 228)$ & $0.56(1126 / 2014)$ & 0.14 & $0.69(198 / 286)$ & $0.68(2087 / 3077)$ & 0.62 \\
\hline Low HDL-cholesterol $^{\mathrm{h}}$ & $0.73(8 / 11)$ & $0.63(86 / 137)$ & 0.51 & $0.56(284 / 503)$ & $0.59(2922 / 4954)$ & 0.27 \\
\hline Hyperglycemia $^{\mathrm{i}}$ & $0.63(48 / 76)$ & $0.60(424 / 708)$ & 0.58 & $0.59(259 / 438)$ & $0.61(2689 / 4383)$ & 0.36 \\
\hline
\end{tabular}

a WC cutoff was set at $88 \mathrm{~cm}$ for men and $82 \mathrm{~cm}$ for women

b The fasting subjects correspond to those shown in Table 1

c Subjects receiving medication for any of hypertension, hyperglycemia, and dyslipidemia were excluded

d Based on the $\chi^{2}$ test

e Defined as systolic blood pressure $\geq 130 \mathrm{mmHg}$ and/or diastolic blood pressure $\geq 85 \mathrm{mmHg}$

${ }^{\mathrm{f}}$ No. of those with WC at and above the cutoff/no. of those with risk factor

g No. of those with WC below the cutoff/no. of those without risk factor

${ }^{\mathrm{h}}$ Defined as HDL-cholesterol $<40 \mathrm{mg} / \mathrm{dL}$

${ }^{\mathrm{i}}$ Defined as hemoglobin A1c $\geq 5.5 \%$

Possible limitations of the present study should be mentioned. First, the diagnostic criteria of MetS, including the WC cutoff, should essentially be determined so that they can effectively predict CVD occurrences. Our findings were not based on this perspective and do not provide information directly relevant to the prevention of CVD. Second, the number of fasting subjects (i.e., the subjects of this study) was limited compared with that of the nonfasting subjects, and if individuals with a certain CVD risk factor (e.g., high DBP) who also had some anthropometric characteristic (e.g., low WC) tended to participate in a fasting state, it could lead to selection bias. To examine this possibility, we compared the fasting and nonfasting subjects in terms of the sensitivity and specificity of WC at the identified cutoffs ( $88 \mathrm{~cm}$ for men and $82 \mathrm{~cm}$ for women) for detecting each of hypertension, low HDL-cholesterol, and hyperglycemia (Table 5), because the above bias would lead to differences in both measurements between the two groups. There were no significant differences between the groups in the sensitivity or specificity of WC for any risk factor (Table 5), suggesting that a substantial effect of selection bias was unlikely. Third, the optimal cutoff of WC for MetS may differ by age [15], although this potential problem has not been considered in any diagnostic criteria. One large cross-sectional study suggested that the optimal WC cutoffs in Japanese men in their $40 \mathrm{~s}, 50 \mathrm{~s}$, and $60 \mathrm{~s}$ were 88,87 , and $85 \mathrm{~cm}$, respectively, as compared with 82,83 , and $83 \mathrm{~cm}$, respectively, for Japanese women [15]. We could not address this issue due to the limited sample size making it difficult to perform precise age-specific analyses. In our study subjects, the prevalence of younger individuals (i.e., those aged 40-49 years) was somewhat higher than that in the general population of Japan or Saga prefecture (data not shown). Moreover, all the study subjects came from a restricted area (Saga city). Therefore, caution must be exercised in generalizing our findings to the Japanese population as a whole.

In conclusion, our ROC analyses demonstrated that the appropriate WC cutoff values for clustering CVD risk factors were $88 \mathrm{~cm}$ for men and $82 \mathrm{~cm}$ for women in the study subjects. Therefore, the current WC cutoff defined by the JCCMS ( $85 \mathrm{~cm}$ for men and $90 \mathrm{~cm}$ for women) appears low for men and too high and insensitive for women in our study population. Our study also suggests that WC represents a measure suitable for detecting the cluster of CVD risk factors, but other anthropometric indices such as BMI may be used as a surrogate of WC, with a properly defined cutoff. Whether the cutoff points of WC and other anthropometric indices are truly appropriate for predicting the risk of CVD should be further examined in longitudinal studies, and the cost-benefit aspects of such cutoff points also warrant further investigations.

Acknowledgments We greatly appreciate the participants and supporting organizations. This study was supported in part by Grantsin-Aid for Scientific Research on the Special Priority Areas of Cancer (No. 17015018) and Innovative Areas (No. 221S0001), Scientific Research (A) (No. 20249038) and (B) (No. 18390182), and Young 
Scientists (A) (No. 20689014) and (B) (No. 18790380) from the Ministry of Education Culture, Sports, Science and Technology, Japan.

Conflict of interest There are no conflicts of interest.

\section{References}

1. Alberti KG, Zimmet PZ. Definition, diagnosis and classification of diabetes mellitus and its complications. Part 1: diagnosis and classification of diabetes mellitus provisional report of a WHO consultation. Diabet Med. 1998;15:539-53.

2. Alberti KG, Zimmet P, Shaw J. Metabolic syndrome-a new world-wide definition. A consensus statement from the International Diabetes Federation. Diabet Med. 2006;23:469-80.

3. Klein S, Allison DB, Heymsfield SB, Kelley DE, Leibel RL, Nonas $\mathrm{C}$, et al. Waist circumference and cardiometabolic risk: a consensus statement from shaping America's health: Association for Weight Management and Obesity Prevention; NAASO, the Obesity Society; the American Society for Nutrition; and the American Diabetes Association. Diabetes Care. 2007;30:1647-52.

4. Matsuzawa Y. Metabolic syndrome-definition and diagnostic criteria in Japan. J Atheroscler Thromb. 2005;12:301.

5. Matsuzawa Y. Metabolic syndrome-definition and diagnostic criteria in Japan. J Jpn Soc Intern Med. 2005;94:188-203. (in Japanese).

6. Japan Society for the Study of Obesity. New criteria for 'obesity disease' in Japan. Circ J. 2002;66:987-92.

7. Oka R, Kobayashi J, Yagi K, Tanii H, Miyamoto S, Asano A, et al. Reassessment of the cutoff values of waist circumference and visceral fat area for identifying Japanese subjects at risk for the metabolic syndrome. Diabetes Res Clin Pract. 2008;79:474-81.

8. Oda E, Watanabe K. Japanese criteria of metabolic syndrome. Circ J. 2006;70:364.

9. Ito H, Nakasuga K, Ohshima A, Maruyama T, Kaji Y, Harada M, et al. Detection of cardiovascular risk factors by indices of obesity obtained from anthropometry and dual-energy X-ray absorptiometry in Japanese individuals. Int J Obes Relat Metab Disord. 2003;27:232-7.

10. Shiwaku K, Anuurad E, Enkhmaa B, Nogi A, Kitajima K, Yamasaki $\mathrm{M}$, et al. Predictive values of anthropometric measurements for multiple metabolic disorders in Asian populations. Diabetes Res Clin Pract. 2005;69:52-62.

11. Hara K, Matsushita Y, Horikoshi M, Yoshiike N, Yokoyama T, Tanaka $\mathrm{H}$, et al. A proposal for the cutoff point of waist circumference for the diagnosis of metabolic syndrome in the Japanese population. Diabetes Care. 2006;29:1123-4.

12. Lee JS, Kawakubo K, Mori K, Akabayashi A. Effective cut-off values of waist circumference to detect the clustering of cardiovascular risk factors of metabolic syndrome in Japanese men and women. Diab Vasc Dis Res. 2007;4:340-5.

13. Miyatake N, Wada J, Matsumoto S, Nishikawa H, Makino H, Numata T. Re-evaluation of waist circumference in metabolic syndrome: a comparison between Japanese men and women. Acta Med Okayama. 2007;61:167-9.

14. Shimajiri T, Imagawa M, Kokawa M, Konami T, Hara H, Kyoku I, et al. Revised optimal cut-off point of waist circumference for the diagnosis of metabolic syndrome in Japanese women and the influence of height. J Atheroscler Thromb. 2008;15:94-9.

15. Narisawa S, Nakamura K, Kato K, Yamada K, Sasaki J, Yamamoto M. Appropriate waist circumference cutoff values for persons with multiple cardiovascular risk factors in Japan: a large cross-sectional study. J Epidemiol. 2008;18:37-42.
16. Matoba $Y$, Inoguchi $T$, Nasu $S$, Suzuki $S$, Yanase T, Nawata H, et al. Optimal cut points of waist circumference for the clinical diagnosis of metabolic syndrome in the Japanese population. Diabetes Care. 2008;31:590-2.

17. Ho SY, Lam TH, Janus ED. Waist to stature ratio is more strongly associated with cardiovascular risk factors than other simple anthropometric indices. Ann Epidemiol. 2003;13:683-91.

18. Lin WY, Lee LT, Chen CY, Lo H, Hsia HH, Liu IL, et al. Optimal cut-off values for obesity: using simple anthropometric indices to predict cardiovascular risk factors in Taiwan. Int $\mathbf{J}$ Obes Relat Metab Disord. 2002;26:1232-8.

19. Hara M, Higaki Y, Imaizumi T, Taguchi N, Nakamura K, Nanri $\mathrm{H}$, et al. Factors influencing participation rate in a baseline survey of a genetic cohort in Japan. J Epidemiol. 2010;20:40-5.

20. Grundy SM, Cleeman JI, Daniels SR, Donato KA, Eckel RH, Franklin BA, et al. Diagnosis and management of the metabolic syndrome: an American Heart Association/National Heart, Lung, and Blood Institute Scientific Statement. Circulation. 2005;112: 2735-52.

21. Executive Summary of the Third Report of the National Cholesterol Education Program (NCEP). Expert panel on detection, evaluation, and treatment of high blood cholesterol in adults (Adult Treatment Panel III). JAMA. 2001;285:2486-97.

22. Hamajima N. The Japan Multi-Institutional Collaborative Cohort Study (J-MICC Study) to detect gene-environment interactions for cancer. Asian Pac J Cancer Prev. 2007;8:317-23.

23. American Diabetes Association. Diagnosis and classification of diabetes mellitus. Diabetes Care. 2008;31(Suppl 1):S55-60.

24. Ito C, Maeda R, Ishida S, Sasaki H, Harada H. Correlation among fasting plasma glucose, two-hour plasma glucose levels in OGTT and HbA1c. Diabetes Res Clin Pract. 2000;50:225-30.

25. Wildman RP, Gu D, Reynolds K, Duan X, He J. Appropriate body mass index and waist circumference cutoffs for categorization of overweight and central adiposity among Chinese adults. Am J Clin Nutr. 2004;80:1129-36.

26. Nguyen TT, Adair LS, He K, Popkin BM. Optimal cutoff values for overweight: using body mass index to predict incidence of hypertension in 18- to 65-year-old Chinese adults. J Nutr. 2008; 138:1377-82.

27. Baik I. Optimal cutoff points of waist circumference for the criteria of abdominal obesity. Circ J. 2009;73(11):2068-75.

28. Han JH, Park HS, Kim SM, Lee SY, Kim DJ, Choi WH. Visceral adipose tissue as a predictor for metabolic risk factors in the Korean population. Diabet Med. 2008;25:106-10.

29. Selvin E, Steffes MW, Zhu H, Matsushita K, Wagenknecht L, Pankow J, et al. Glycated hemoglobin, diabetes, and cardiovascular risk in nondiabetic adults. N Engl J Med. 2010;362:800-11.

30. Hsieh SD, Yoshinaga H. Waist/height ratio as a simple and useful predictor of coronary heart disease risk factors in women. Intern Med. 1995;34:1147-52.

31. Lee JS, Aoki K, Kawakubo K, Gunji A. A study on indices of body fat distribution for screening for obesity. J Occup Health. 1995;37:9-18.

32. Hsieh SD, Yoshinaga H, Muto T. Waist-to-height ratio, a simple and practical index for assessing central fat distribution and metabolic risk in Japanese men and women. Int $\mathrm{J}$ Obes Relat Metab Disord. 2003;27:610-6.

33. Han TS, McNeill G, Seidell JC, Lean ME. Predicting intraabdominal fatness from anthropometric measures: the influence of stature. Int J Obes Relat Metab Disord. 1997;21:587-93.

34. WHO Expert Committee. Physical status: the use and interpretation of anthropometry. World Health Organ Tech Rep Ser. 1995;854:312-44.

35. WHO Expert Consultation. Appropriate body-mass index for Asian populations and its implications for policy and intervention strategies. Lancet. 2004;363:157-63. 